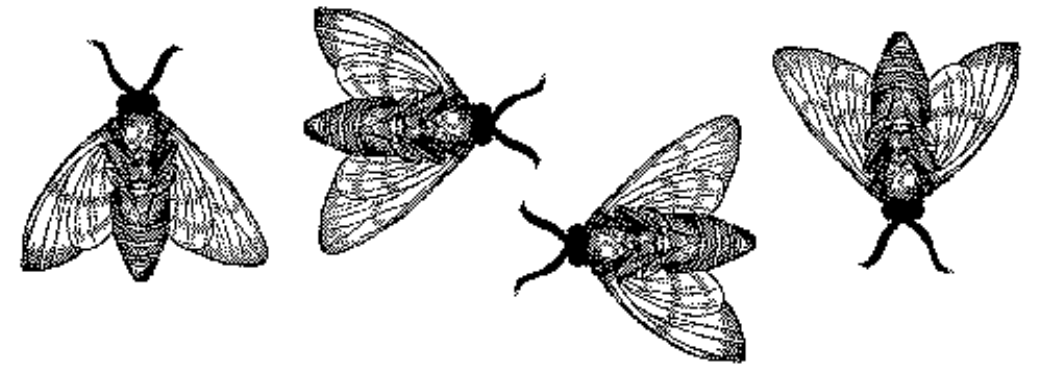


a. 



\section{Compression of Video Tracking and Bandwidth Balancing Routing in Wireless Multimedia Sensor Networks}

\author{
Yin Wang School of \\ Computer Science \\ Southwest Petroleum \\ University \\ Chengdu, Sichuan 610500, \\ China \\ ywang12@ltu.edu
Juan Guo Department of Computer Science and
Information Systems
University of North Georgia
juan.guo@ung.edu

\author{
Jianjun Yang Department of \\ Computer Science and \\ Information Systems \\ University of North Georgia \\ jianjun.yang@ung.edu
}

\author{
Ju Shen Department of \\ Computer Science \\ University of Dayton \\ jshen1@udayton.edu
}

\begin{abstract}
There has been a tremendous growth in multimedia applications over wireless networks. Wireless Multimedia Sensor Networks(WMSNs) have become the premier choice in many research communities and industry. Many state-of-art applications, such as surveillance, traffic monitoring, and remote heath care are essentially video tracking and transmission in WMSNs. The transmission speed is constrained by big size of video data and fixed bandwidth allocation in constant routing path. In this paper, we present a CamShift based algorithm to compress the tracking of videos. Then we propose a bandwidth balancing strategy in which each sensor node is able to dynamically select the node for next hop with the highest potential bandwidth capacity to resume communication. Key to the strategy is that each node merely maintains two parameters that contains its historical bandwidth varying trend and then predicts its near future bandwidth capacity. Then forwarding node selects the next hop with the highest potential bandwidth capacity. Simulations demonstrate that our approach significantly increases the data received by sink node and decreases the delay on video transmission in Wireless Multimedia Sensor Network environment.
\end{abstract}

\section{Keywords}

CamShift, bandwidth balancing, network traffic

\footnotetext{
*Permanent address of Yin Wang: Lawrence Technological University, Southfield, Michigan 48075, USA. This work was conducted when Yin Wang was visiting the Southwest Petroleum University.
}

\author{
Kun Hua Department of \\ Electrical and Computer \\ Engineering \\ Lawrence Technological \\ University \\ khua@ltu.edu
}

\section{INTRODUCTION}

Wireless Multimedia Sensor Networks(WMSNs) have emerged as one of the key technologies for wireless communications. They are undergoing rapid development and have inspired numerous applications because of their advantages. More recently, the availability of inexpensive hardware such as CMOS cameras and microphones that are able to ubiquitously capture multimedia content from the environment has fostered the development of WMSNs, which are networks of wirelessly interconnected devices that allow retrieving video and audio streams, still images, and scalar sensor data 1 .

Wireless multimedia sensor networks will enable several new applications [2], which include: First, Surveillance.Video and audio sensors will be used to enhance and complement existing surveillance systems against crime and terrorist attacks. Large scale networks of video sensors can extend the ability of law-enforcement agencies to monitor areas, public events, private properties, and borders. Second, Traffic Monitoring and Enforcement.It will be possible to monitor car traffic in big cities or highways and deploy services that offer traffic routing advice to avoid congestion.Third, Personal and Health Care. Multimedia sensor networks can be used to monitor and study the behavior of people as a means to identify the causes of illnesses. Fourth, Environmental and Industrial.Several projects on habitat monitoring that use acoustic and video feeds are being envisaged, in which information has to be conveyed in a time-critical fashion.

Despite of the fact that wireless bandwidth has been increasing significantly, from a theoretical limit of $11 \mathrm{Mbps}$ for $802.11 \mathrm{~b}$ to $54 \mathrm{Mbps}$ for $802.11 \mathrm{~g}$, and to $540 \mathrm{Mbps}$ for $802.11 n$, there has always been high bandwidth demand resulting from multimedia data 3 . Moreover, the routing path from a node to the sink node is normally created by a kind of routing protocol and the path is fixed. Hence some nodes are affording busy traffic but other nodes that are not in the routing path are idle. For video tracking, video data is frequently transferred over network. It is extremely important to compress video data and find best transmission 
strategy. In this paper, we developed a novel compression video tracking and bandwidth balancing mechanism to boost the video transmission speed in WMSNs. Our contributions are two folds. First, we present a CamShift based algorithm to compress tracking of videos. Then we propose a bandwidth balancing strategy in which each sensor node is able to dynamically select the node for next hop with the highest potential bandwidth capacity to resume communication. Our approach significantly increase the data received by sink node and decrease the latency on video transmission in Wireless Multimedia Sensor Network environment.

The rest of the paper is organized as follows. Section II discusses the related research on this topic. Section III proposes a novel method that select the best relay station. We evaluate the proposed schemes by simulations and describe the performance results in Section IV. Section V concludes the paper.

\section{RELATED WORK}

Various approaches regarding video processing [12, 13, 14, 15] and transmission [9, 10, 11, 18, 19] over WMSNs were proposed. Q. Cai et al. 23, 24 proposed a dynamic action recognition, which is important to capture videos in WMSNs. In their approach, a Dynamic Structure Preserving Map is proposed to effectively recognize objects' actions in video sequences. They modified and improved the adaptive learning procedure in self-organizing map (SOM) to capture dynamics of best matching neurons through Markov random walk. The method can learn implicit spatial-temporal correlations from sequential action feature sets and preserve the intrinsic topologies characterized by different human motions. The mechanism is able to learn low-level features in challenging video data. The projection from high dimensional action features to low dimensional latent neural distribution significantly reduces the computational cost and data redundancy in the recognition process.

Ahmed et al. 27] studied improving the quality of MPEG-4 transmission on wireless using Differentiated Services. They investigated QoS provisioning between MPEG-4 video application and Diffserv networks. To achieve the best possible QoS, all the components involved in the transmission process must collaborate. For example, the server must use stream properties to describe the QoS requirement for each stream to the network. They propose a solution by distinguishing the video data into important one and less important one. Packets marked as less important are dropped in the first case if there is any congestion, so that the receiver can regenerate the video with the received important information.

Budagavi et al. 28, improved the performance of video over wireless channels by multiframe video coding. The multiframe coder uses the redundancy that exists across multiple frames in a typical video conferencing sequence so that additional compression can be achieved using their MF-BMC (Multi Frame - Block Motion Compensation) approach. They modeled the error propagation using the Markov chain, and concluded that use of multiple frames in motion increases the robustness. Their proposed MF-BMC scheme has been shown to be more robust on wireless networks when compared to the base-level H.263 codec which uses SF-BMC (Single Frame-BMC).
J. Zhang et al. 4, 5] developed a wildlife monitoring system based on wireless image sensor networks. In their study, architecture of the wildlife monitoring system based on the wireless image sensor networks was presented to overcome the shortcomings of the traditional monitoring methods. Specifically, some key issues including design of wireless image sensor nodes and software process development have been studied and presented. A self-powered rotatable wireless infrared image sensor node and an aggregation node designed for large amounts of data were developed. In addition, their corresponding software was designed. The proposed system is able to monitor wildlife accurately, automatically, and remotely in all-weather condition, which lays foundations for applications of wireless image sensor networks in wildlife monitoring.

Q. Sun et al. proposed wireless sensor monitoring systems 20, 21, 22. Compared with conventional image and video based monitoring systems, wireless sensor monitoring systems is an alternative technology for pattern recognition in multimedia field due to its low cost, low data throughput, self-management, high robustness under various conditions. Nowadays, such wireless sensor based sensing systems have been widely investigated, especially in human sensing. Specifically, among various wireless sensors, pyroelectric infrared (PIR) sensor and fiber-optic sensor are the most popular ones in human tracking, human identification, and activity recognition fields. Due to the low data throughput property of wireless sensors, the most challenging issues are efficient information acquisition scheme design and light-weight learning algorithm development. A classical work for information acquisition design with a geometric sampling structure and a pseudo-random visibility modulation is created. Such design can effectively reduce the effects of occlusion and energy variation, thus, enhance information acquisition. Another milestone work proposed a space encoding scheme to avoid the ambiguity generated by bipedal movements of humans. This scheme is able to dramatically reduce the false alarm rate in human localization for fiberoptic sensors. With respect to light-weight learning algorithm design, presented a Bayesian networks and region of interests (ROI) based reasoning method. This method can be applied to any sensor modalities.

J. Campbell et al. 29] presented IRISNET, a sensor network architecture that enables the creation of a planetary-scale infrastructure of multimedia sensors that can be shared by a large number of applications. To ensure the efficient collection of sensor readings, IRISNET enables the applicationspecific processing of sensor feeds on the significant computation resources that are typically attached to multimedia sensors. IRISNET enables the storage of sensor readings close to their source by providing a convenient and extensible distributed XML database infrastructure. Finally, IRISNET provides a number of multimedia processing primitives that enable the effective processing of sensor feeds in-network and at-sensor.

S. Shen et al. developed new sensing technologies based on nanostructured devices, which significantly reduced the physical size and weight of sensor nodes and therefore promoted the flexible and cost-effective deployment of wireless sensor networks [6, 7, 8]. 


\section{PROBLEM FORMULATION}

To boost the transmission speed of video data over WMSNs, our mechanism works on two folds. One is the video tracking and compression. The second is bandwidth balancing routing.

\subsection{Video tracking and compression}

We present CamShift based tracking algorithm based on Histogram Back-projection 30] and Mean Shift Algorithm 31. The mean shift algorithm works well on static probability distributions but not on dynamic ones. Our approach detects the peak in the probability distribution image by applying mean shift while handling dynamic distributions by readjusting the search window size for the next frame based on the 0th moment of the current frames distribution, which allows the algorithm to anticipate object movement to quickly track the object in the next scene. Specifically, the search area can be restricted around the last known position of the target, resulting in possibly large computational savings. In a single image, the process is iterated until convergence an upper bound on the number of iterations is reached. The detection algorithm is applied to successive frames of a video sequence to track target. This type of scheme introduces a feed-back loop, in which the result of the detection is used as input to the next detection process.

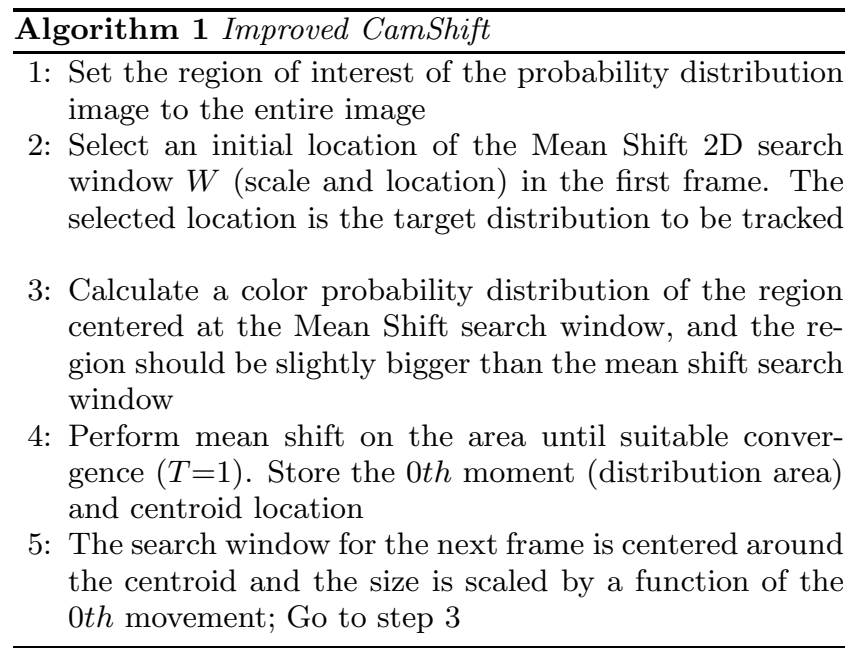

In algorithm 1, we choose 1.0 as the threshold of convergence to get a satisfying tracking result. The location of the search window is used to help track movement.

\subsection{Bandwidth Balancing Routing}

In WMSNs, sensor nodes collect video information, process video, and transmit data to sink node(base station). The sink nodes then sends data to Internet. Fig. 1 shows an instance of WMSNs, in which sensor nodes handle video data and transfer the data to the sink node, then to Internet. The communications over nodes are based on channels. Channel bandwidth is critical for transmission speed. We define the residual bandwidth ability(RBA) for a node as its possible bandwidth deducting the real bandwidth over its channel. For Instance, if the possible bandwidth for a node is $100 \mathrm{MB} / \mathrm{S}$, the traffic occupies $60 \mathrm{MB} / \mathrm{S}$, then its $\mathrm{RBA}$ is $40 \mathrm{MB} / \mathrm{S}$. We use a simple case to illustrate our main idea. Suppose node a collects video data and got it processed.
Then it intends to send the data to the sink node. The routing path can be set up by any routing algorithm. Normally, it is fixed when it is set up. The path a-b-c-d can be a routing path. If many other nodes also send data to $b$, and then to the sink node, $b$ has to afford a lot of traffic, while $e$ or $f$ have low traffic. Considering this scenario, we are thinking about if node $a$ can select one of its neighbors with highest RBA as the next hop. It may not be $b$.

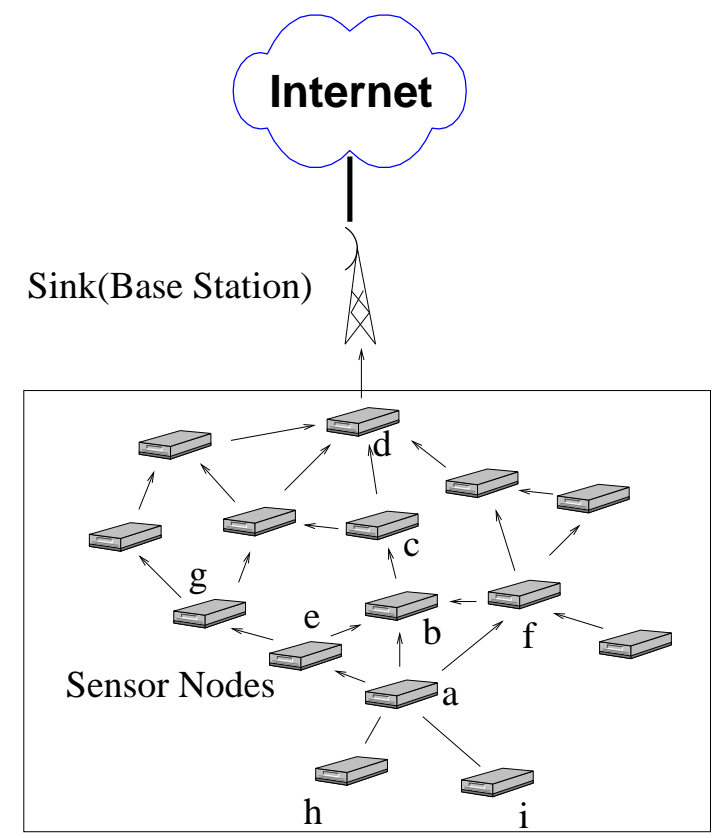

Figure 1: An example of WMSN

We further assume that the historical RBA vector up to this moment of $b$ is $[\ldots, 85,70,55,40], e$ is $[\ldots, 25,32,35,39]$, and $f$ is $[\ldots, 50,46,42,38]$. A simple strategy will let $a$ select $b$ because it has the highest RBA at the moment. Seemingly, $b$ is the best choice. However, since $b$ is a hot node serving a high volume traffic and its RBA is going down dramatically, while $e$ or $f$ is serving less traffic and their RBA are going down slowly or even going up, so $a$ should select $e$ or $f$ to avoid hot node and then balance bandwidth utilization.

Our goal is to let each node select the node of next hop with highest potential RBA. In our approach, each candidate node can predict its potential RBA for next time based on its historical RBA and current RBA. Then the forwarding node selects the best candidate. In this example, our strategy will let $a$ choose $e$.

\subsection{Calculation of Residual Bandwidth Abil- ity}

The communications between nodes in a Wireless Multimedia Sensor Network vary from time to time. It is critical for a node to forward data to the possible best node. For each node, the trend of its bandwidth's changing is essential to calculate its potential RBA. Suppose the current time is $k$. A naive method to calculate its potential RBA is this: Let node $N_{i}$ save all its historical and current (time $k$ ) RBA $R_{i_{0}}$, $R_{i_{1}}, \ldots, R_{i_{k}}$ in a vector. Then it can calculate its potential 
RBA for time $k+1$ by curve-fitting in numerical analysis that approximates its RBA changing trend. The limitations of this method are to spend too much space to save the RBA values in the vector and too complex for curve-fitting computation.

We propose a statistical based strategy to let each node predict its future RBA. In this scheme, each node only maintains two parameter values. One is its trend of RBA acceleration and the second is the variance of the acceleration. The two parameters store how its RBA changes. Together with the node's current bandwidth, its future bandwidth capacity is achieved by predicted RBA(potential RBA). Thus, each node is able to figure out the best next hop subsequently.

We define the following notations to represent the terms regarding our approach for node $N_{i}$.

$R_{i_{k}}$ : The measured residual bandwidth at time $k$.

$\hat{R_{i_{k}}}$ : The bandwidth predicted at time $k$ (Potential RBA).

$a_{i_{k}}$ : The acceleration measured at time $k$. It indicates how RBA changes during a time slot.

$\hat{a_{i_{k}}}$ : The acceleration at time $k$ evolved from time $k-1$.

$a_{i_{k}}$ : The potential acceleration at time $k$.

$v_{i_{k}}$ : The variance of acceleration updated at time $k$.

$v_{i_{k}}^{-}$: The variance of acceleration at time $k$ evolved from time $k-1$.

$\epsilon$ : The error or noise in the process.

$B_{i_{k}}$ : The blending factor at time $k$.

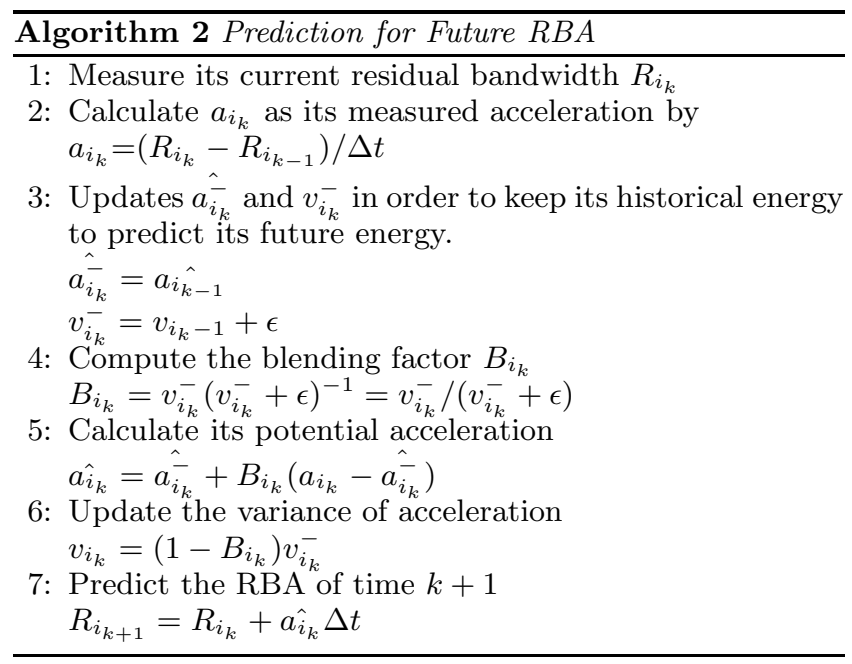

At time $k$, node $N_{i}$ calls algorithm 2 to calculate future RBA. $N_{i}$ knows its maintained values $\hat{a_{i_{k-1}}^{\hat{n}}}$ and $v_{i_{k-1}}$. It measures its $R_{i_{k}}$ in step 1 . Then it calculates measured acceleration in step 2. $N_{i}$ updates $\hat{a_{i_{k}}}$ and $v_{i_{k}}^{-}$in order to keep its historical RBA to predict its future RBA in step 3. $N_{i}$ also computes the blending factor $B_{i_{k}}$ in step 4 , which indicates how much the acceleration changes from last time to current time. Once $N_{i}$ obtains the blending factor $B_{i_{k}}$ and the evolved acceleration $\hat{a_{i_{k}}}$, it knows how much the acceleration changes with evolved acceleration. Additionally, $R_{i}$ considers the measured acceleration $a_{i_{k}}$. Then it calculates its potential acceleration in step 5. This acceleration will be used to predict its RBA of time $k+1$. It updates the variance of acceleration for future utilization in step 6 and predicts its future RBA of time $k+1$ in step 7 .

\subsection{Region for next hop}

When a node $N_{i}$ intends to transfer video data to the sink node, it selects the neighbor node with highest potential RBA as its next hop. Apparently, $N_{i}$ will not select any nodes in the opposite direction from $N_{i}$ to the sink node.

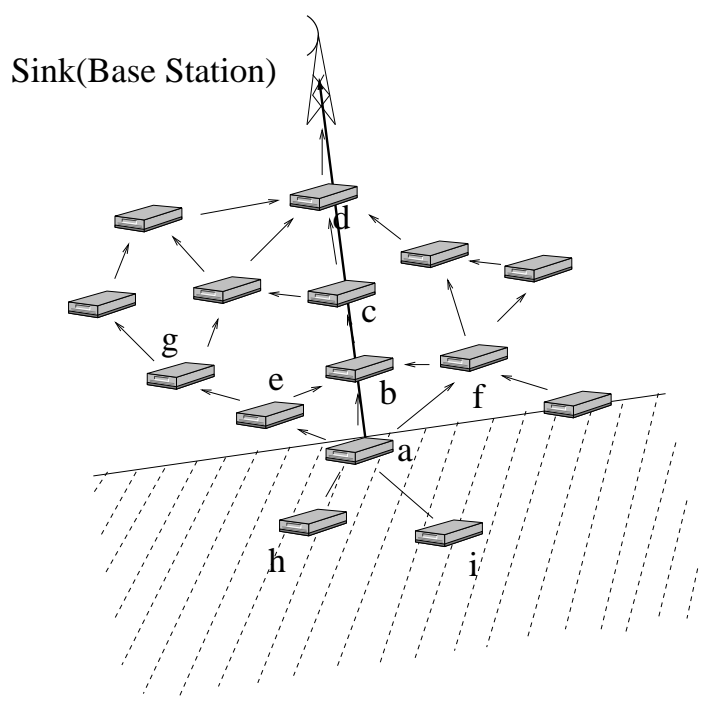

Figure 2: Region for next hop

How does node $N_{i}$ figure out the region where next hop falls? In current WMSNs, each device is equipped with GPS and hence it knows its location. We assume that the sender knows its location and the location of the receiver. The assumption is very common in geographic routing [16, 17. Fig. 2 shows the scenario. Suppose node $a$ intends to send video data to the sink node, it creates a ray by connecting itself with the sink. Then it draws a line perpendicular to the ray. Then the region on the same side of the sink is available region for next hop. As in Fig. 2 $a$ will never select a node in the dash line region for next hop.

\subsection{Bandwidth Balancing Routing}

Once a node $N_{i}$ intends to send video data to the sink, it needs to choose the node with highest RBA as the next hop. It sends a signal to its neighbors in the available region. Upon receiving the signal, each receiver calls algorithm 2 to calculate its RBA of next time and returns the RBA to node $N_{i}$. When node $N_{i}$ receives all RBAs, it selects the one with highest RBA as the next hop.

\section{PERFORMANCE EVALUATION}

We evaluated our mechanism in a simulated noiseless radio network environment by MATLAB. We create a topology that consists of a number of randomly distributed nodes. A sink node is located in the edge of the area. Our approach is based on balancing bandwidth utilization by RBA selection.We compare our approach("RBA") with LEACH 25 and GPSR 26 in terms of data received by sink node and 
number of alive nodes. To evaluate the first item, we performed a sequence of experiments in which the number of nodes varies from 50 to 800 with increment of 50 . For each number of mobile users, we measure size of data the sink node received 10 times and present the average.

Fig. 3 shows our evaluation of data received in sink node. Among the three approaches, the scheme GPSR results in the lowest received data. Our approach RBA generate highest received data. This indicates that bandwidth balancing approach can improve aggregate data for the sink because the bandwidths are allocated more reasonable over the network.

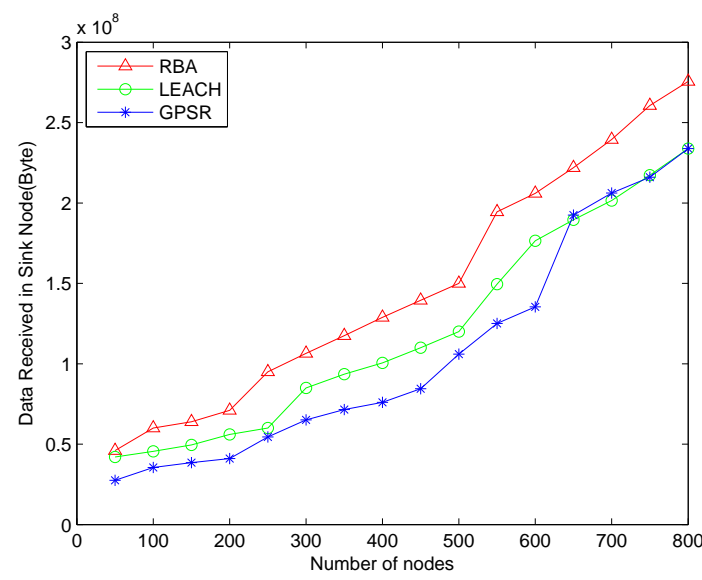

Figure 3: Data received by sink node

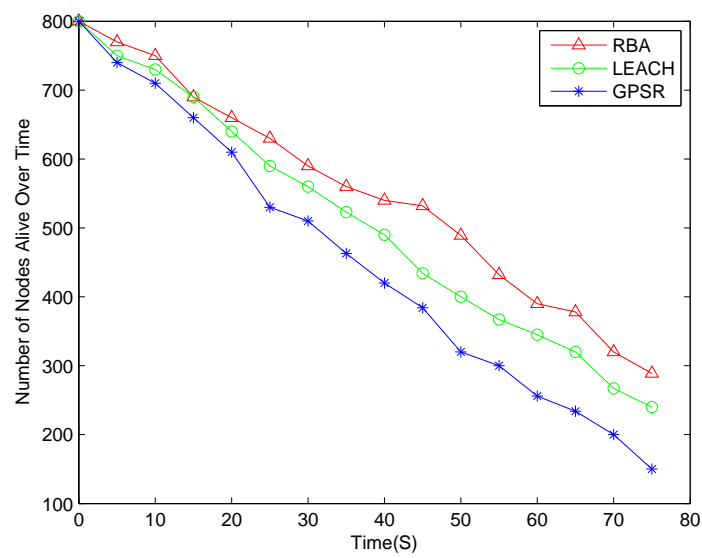

Figure 4: Number of alive nodes

In WMSNs, the life time of a relay station is important. If one node runs turns down, there will be a hole [16, 17, 26] and the network performance will be decreased dramatically. So we also measure how many alive nodes along with the time elapsing. We initialize 800 nodes in the environment and observe how many alive nodes every 5 seconds up to $80 \mathrm{th}$ second. Fig. 4 shows how many alive nodes of the three schemes with time elapsing. GPSR results in the lowest average lift time. That is because GPSR adopts a landmark node to forward a lot of data when there is a hole. However, the landmark is easy to turn down with high volume traffic. Then the hole is bigger [16, 17]. Our approach maintains the largest alive nodes because we always balance bandwidth and then makes the life time longer.

\section{CONCLUSION}

In this paper, we present a RBA based bandwidth balancing mechanism to transfer video data in Wireless Multimedia Sensor Networks. We first present a CamShift based approach to compress video for video tracking and transmission. Then we propose a statistical based mechanism to balance bandwidth utilization.In the approach, each node only needs to remain two factors to store its historical residual bandwidth ability(RBA) trend and then it is able to predict its bandwidth of near future. Each node selects the next hop with potential highest RBA to relay data transmission. Simulations demonstrate that our approach generates highest received data to sink node in the network and largest alive nodes over time elapsing.

\section{REFERENCES}

[1] Akyildiz, Ian F., Tommaso Melodia, and Kaushik R. Chowdhury. A survey on wireless multimedia sensor networks. Computer networks, 1.4 (2007): 921-960.

[2] Akyildiz, Ian F., Tommaso Melodia, and Kaushik R. Chowdhury. Wireless multimedia sensor networks: Applications and testbeds. Proceedings of the IEEE, 96.10 (2008): 1588-1605.

[3] Nguyen Dong, Thinh Nguyen, and Xue Yang. Multimedia wireless transmission with network coding. Packet Video, 2007. IEEE, 2007.

[4] Junguo Zhang, Xin Luo, Chen Chen, Zhen Liu, and Shuai Cao. A wildlife monitoring system based on wireless image sensor networks. Sensors $\&$ Transducers, 2014. volume 180, number 10, pages $=104-109$.

[5] J. Zhang, W. Li, X. Zhao, X. Bai, and C. Chen. Simulation and Research on Data Fusion Algorithm of the Wireless Sensor Network Based on NS2. 2009 WRI World Congress on Computer Science and Information Engineering, Los Angeles, CA, March 2009, pp. 66-70.

[6] Shen, S., V. DeGeorge, P. R. Ohodnicki, S. J. Kernion, V. Keylin, J. F. Huth, and M. E. McHenry. Induced anisotropy in FeCo-based nanocomposites: Early transition metal content dependence. Journal of Applied Physics, 115 (17), 17A335 (2014).

[7] Shen, S., P. R. Ohodnicki, S. J. Kernion, and M. E. McHenry. Two-current model of the composition dependence of resistivity in amorphous (Fe100- xCox) 89- yZr7B4Cuy alloys using a rigid-band assumption. Journal of Applied Physics, 112, no. 10 (2012): 103705.

[8] Shen, Shen, Paul R. Ohodnicki, Samuel Kernion, Alex Leary, Vladimir Keylin, Joseph Huth, and Michael E. McHenry. Nanocomposite alloy design for high frequency power conversion applications. TMS Annual, 2012 (2012).

[9] Xu, Kunjie, David Tipper, Prashant Krishnamurthy, and Yi Qian. An efficient hybrid model and dynamic performance analysis for multihop wireless networks. in Computing, Networking and Communications (ICNC), 2013 International Conference on, pp. 1090-1096. IEEE, 2013. 
[10] Xu, Kunjie Xu, Zhou, Mu. Energy balanced chain in ieee 802.15. 4 low rate wpan. in Computing, Networking and Communications (ICNC), 2013 International Conference on. IEEE, 2013, pp. 1010Ü 1015.

[11] Xu, Kunjie , Tipmongkonsilp, Siriluck , Tipper, David , Qian, Yi , and Krishnamurthy, Prashant. A time dependent performance model for multi-hop wireless networks with cbr traffic. in 29th IEEE International Performance Computing and Communications Conference, 2010.

[12] Shen, Ju, and Sen-Ching S. Cheung. Layer depth denoising and completion for structured-light rgb-d cameras. In Computer Vision and Pattern Recognition (CVPR), 2013 IEEE Conference on, pp. 1187-1194. IEEE, 2013.

[13] Shen, Ju, Po-Chang Su, Sen-ching Samson Cheung, and Jian Zhao. Virtual mirror rendering with stationary rgb-d cameras and stored 3-d background. Image Processing, IEEE Transactions, on 22, no. 9 (2013): 3433-3448.

[14] Shen, Ju, Anusha Raghunathan, Sen-ching S. Cheung, and Rita Patel. Automatic content generation for video self modeling. In Multimedia and Expo (ICME), 2011 IEEE International Conference on, pp. 1-6. IEEE, 2011.

[15] Yang, Jianjun, Wang, Yin, Wang, Honggang, Hua, Kun, Wang, Wei and Shen, Ju. Automatic Objects Removal for Scene Completion. the 33rd Annual IEEE International Conference on Computer Communications (INFOCOM'14), Workshop on Security and Privacy in Big Data, Toronto, Canada, April 2014.

[16] Yang, Jianjun, and Zongming Fei. HDAR: Hole detection and adaptive geographic routing for ad hoc networks. In Computer Communications and Networks (ICCCN), 2010 Proceedings of 19th International Conference on, pp. 1-6. IEEE, 2010.

[17] Yang, Jianjun, and Zongming Fei. ITGR: intermediate target based geographic routing. In Computer Communications and Networks (ICCCN), 2010 Proceedings of 19th International Conference on, pp. 1-6. IEEE, 2010.

[18] Yang, Jianjun, and Zongming Fei. Broadcasting with prediction and selective forwarding in vehicular networks. International journal of distributed sensor networks, 2013 (2013).

[19] Yang, Jianjun, and Zongming Fei. Bipartite graph based dynamic spectrum allocation for wireless mesh networks. In Distributed Computing Systems Workshops, 2008. ICDCS'08. 28th International Conference on, pp. 96-101. IEEE, 2008.

[20] Sun, Qingquan, Yu, Weihong, Kochurov, Nikolai, Hao, Qi , and Hu, Fei. Dspm: A Multi-Agent-Based Intelligent Sensor and Actuator Network Design for Smart House and Home Automation. Journal of Sensor and Actuator Networks, 2, no. 3 (2013): 557-588.

[21] Sun, Qingquan, Fei, Hu, and Hao, Qi. Context awareness emergence for distributed binary pyroelectric sensors. In Multisensor Fusion and Integration for Intelligent Systems (MFI), 2010 IEEE Conference on, pp. 162-167. IEEE, 2010.

[22] Hu, Fei, Sun, Qingquan, and Hao, Qi. Mobile targets region-of-interest via distributed pyroelectric sensor network: Towards a robust, real-time context reasoning. In Sensors, 2010 IEEE, pp. 1832-1836. IEEE, 2010.

[23] Cai, Qiao, Yafeng Yin, and Hong Man. Dspm: Dynamic structure preserving map for action recognition. Multimedia and Expo (ICME), 2013 IEEE International Conference on. IEEE, 2013.

[24] Cai, Qiao, Yafeng Yin, and Hong Man. Learning spatio-temporal dependencies for action recognition. ICIP, 2013.

[25] Heinzelman, Wendi Rabiner, Anantha Chandrakasan, and Hari Balakrishnan. Energy-efficient communication protocol for wireless microsensor networks. In System Sciences, 2000. Proceedings of the 33rd Annual Hawaii International Conference on, pp. 10-pp. IEEE, 2000..

[26] Karp, Brad, and Hsiang-Tsung Kung. GPSR: Greedy perimeter stateless routing for wireless networks. In Proceedings of the 6th annual international conference on Mobile computing and networking, pp. 243-254. ACM, 2000.

[27] Ahmed, Toufik, Ahmed Mehaoua, and Guillaume Buridant. Implementing MPEG-4 video on demand over IP differentiated services. Global Telecommunications Conference, 2001. GLOBECOM'01. IEEE. Vol. 4. IEEE, 2001.

[28] Budagavi, Madhukar, and Jerry D. Gibson. Multiframe video coding for improved performance over wireless channels. Image Processing, IEEE Transactions, 10.2 (2001): 252-265.

[29] Campbell, Jason, et al. Irisnet: an internet-scale architecture for multimedia sensors. Proceedings of the 13 th annual ACM international conference on Multimedia, ACM, 2005.

[30] Lee, Jae-Yeong, and Wonpil Yu. Visual tracking by partition-based histogram backprojection and maximum support criteria. Robotics and Biomimetics (ROBIO), 2011 IEEE International Conference on. IEEE, 2011.

[31] Yang, Changjiang, Ramani Duraiswami, and Larry Davis. Efficient mean-shift tracking via a new similarity measure. Computer Vision and Pattern Recognition, 2005. CVPR 2005. IEEE Computer Society Conference on. Vol. 1. IEEE, 2005.

[32] M. Bowman, S. K. Debray, and L. L. Peterson. Reasoning about naming systems. ACM Trans. Program. Lang. Syst., 15(5):795-825, November 1993.

[33] J. Braams. Babel, a multilingual style-option system for use with latex's standard document styles. TUGboat, 12(2):291-301, June 1991.

[34] M. Clark. Post congress tristesse. In TeX90 Conference Proceedings, pages 84-89. TeX Users Group, March 1991.

[35] M. Herlihy. A methodology for implementing highly concurrent data objects. ACM Trans. Program. Lang. Syst., 15(5):745-770, November 1993.

[36] L. Lamport. LaTeX User's Guide and Document Reference Manual. Addison-Wesley Publishing Company, Reading, Massachusetts, 1986.

[37] S. Salas and E. Hille. Calculus: One and Several Variable. John Wiley and Sons, New York, 1978. 


\section{Compression of Video Tracking and Bandwidth Balancing Routing in Wireless Multimedia Sensor Networks}

\author{
Yin Wang Department of \\ Mathematics and Computer \\ Science \\ Lawrence Technological \\ University \\ ywang12@ltu.edu
Juan Guo Department of Computer Science and Information Systems
University of North Georgia juan.guo@ung.edu

\author{
Jianjun Yang Department of \\ Computer Science and \\ Information Systems \\ University of North Georgia \\ jianjun.yang@ung.edu
}

\author{
Ju Shen Department of \\ Computer Science \\ University of Dayton \\ jushen.tom@gmail.com
}

\begin{abstract}
There has been a tremendous growth in multimedia applications over wireless networks. Wireless Multimedia Sensor Networks(WMSN) have become the premier choice in many research communities and industry. Many state-to-art applications, such as surveillance, traffic monitoring, and remote heath care are essentially video tracking and transmission in WMSN. The transmission speed is constrained by big size of video data and fixed bandwidth allocation in fixed routing path. In this paper, we present a CamShift algorithm to compress the tracking of videos. Then we propose a bandwidth balancing strategy in which the sensor nodes are able to dynamically select the node for next hop with the highest potential bandwidth capacity to resume communication. Key to the strategy is that each node merely maintains two parameters that contains the historical network traffic and then calculates its bandwidth capacity. Then each node selects the next hop with the highest potential bandwidth capacity. Simulations demonstrate that our approach significantly increase the aggregate throughput and decrease the latency on video transmission in Wireless Multimedia Sensor Network environment.
\end{abstract}

\section{Keywords}

CamShift, bandwidth balancing, network traffic

\section{INTRODUCTION}

Wireless Multimedia Sensor Networks(WMSN) have emerged as one of the key technologies for wireless communications. They are undergoing rapid development and have inspired numerous applications because of their advantages. More

\author{
Kun Hua Department of \\ Electrical and Computer \\ Engineering \\ Lawrence Technological \\ University \\ khua@ltu.edu
}

recently, the availability of inexpensive hardware such as CMOS cameras and microphones that are able to ubiquitously capture multimedia content from the environment has fostered the development of Wireless Multimedia Sensor Networks (WMSNs), which are networks of wirelessly interconnected devices that allow retrieving video and audio streams, still images, and scalar sensor data[?].

Wireless multimedia sensor networks will enable several new applications[?], which include: First, Surveillance.Video and audio sensors will be used to enhance and complement existing surveillance systems against crime and terrorist attacks. Large scale networks of video sensors can extend the ability of law-enforcement agencies to monitor areas, public events, private properties, and borders. Second, Traffic Monitoring and Enforcement.It will be possible to monitor car traffic in big cities or highways and deploy services that offer traffic routing advice to avoid congestion.Third, Personal and Health Care. Multimedia sensor networks can be used to monitor and study the behavior of people as a means to identify the causes of illnesses. Fourth, Environmental and Industrial.Several projects on habitat monitoring that use acoustic and video feeds are being envisaged, in which information has to be conveyed in a time-critical fashion.

Despite of the fact that wireless bandwidth has been increasing significantly, from a theoretical limit of $11 \mathrm{Mbps}$ for $802.11 \mathrm{~b}$ to $54 \mathrm{Mbps}$ for $802.11 \mathrm{~g}$, and to $540 \mathrm{Mbps}$ for $802.11 \mathrm{n}$, there has always been high bandwidth demand resulting from multimedia data[?]. Moreover, the routing path from a node to the sink node is normally created by a kind of routing protocol and the path is fixed. Hence some nodes are affording busy traffic but other nodes that are not in the routing path are idle. In this paper, we developed a novel compression video tracking and bandwidth balancing mechanism to boost the video transmission speed in WMSNs. Our contributions are two folds. First, we present a CamShift algorithm to compress tracking of videos. Then we propose a bandwidth balancing strategy in which the sensor nodes are able to dynamically select the node for next hop with the highest potential bandwidth capacity to re- 
sume communication. Key to the strategy is that each node merely maintains two parameters that contains the historical network traffic and then calculates its bandwidth capacity. Then each node selects the next hop with the highest potential bandwidth capacity. Our approach significantly increase the aggregate throughput and decrease the latency on video transmission in Wireless Multimedia Sensor Network environment.

The rest of the paper is organized as follows. Section II discusses the related research on this topic. Section III proposes a novel method that select the best relay station. We evaluate the proposed schemes by simulations and describe the performance results in Section IV. Section V concludes the paper.

\section{RELATED WORK}

Various approaches regarding video processing and transmission over WMSNs were proposed. Q. Cai et al. [?, ?] proposed a dynamic action recognition, which is important to capture videos in WMSNs. In their approach, a Dynamic Structure Preserving Map is proposed to effectively recognize objects' actions in video sequences. They modified and improved the adaptive learning procedure in self-organizing map (SOM) to capture dynamics of best matching neurons through Markov random walk. The method can learn implicit spatial-temporal correlations from sequential action feature sets and preserve the intrinsic topologies characterized by different human motions. The mechanism is able to learn low-level features in challenging video data. The projection from high dimensional action features to low dimensional latent neural distribution significantly reduces the computational cost and data redundancy in the recognition process.

Ahmed et al. [?] studied to improve the quality of MPEG-4 transmission on wireless using Differentiated Services. They investigated QoS provisioning between MPEG-4 video application and Diffserv networks. To achieve the best possible QoS, all the components involved in the transmission process must collaborate. For example, the server must use stream properties to describe the QoS requirement for each stream to the network. They propose a solution by distinguishing the video data into important video data and less important video data. Packets marked as less important are dropped in the first case if there is any congestion, so that the receiver can regenerate the video with the received important information.

Budagavi et al. [?] improved the performance of video over wireless channels by multiframe video coding. The multiframe coder uses the redundancy that exists across multiple frames in a typical video conferencing sequence so that additional compression can be achieved using their MF-BMC (Multi Frame - Block Motion Compensation) approach. They modeled the error propagation using the Markov chain, and concluded that use of multiple frames in motion increases the robustness. Their proposed MF-BMC scheme has been shown to be more robust on wireless networks when compared to the base-level H.263 codec which uses SF-BMC (Single Frame-BMC).

J. Zhang et al.[?, ?] developed a wildlife monitoring system based on wireless image sensor networks. In their study, system architecture of the wildlife monitoring system based on the wireless image sensor networks was presented to overcome the shortcomings of the traditional monitoring methods. Specifically, some key issues including design of wireless image sensor nodes and software process design have been studied and presented. A self-powered rotatable wireless infrared image sensor node and an aggregation node designed for large amounts of data were developed. In addition, their corresponding software was designed. The proposed system is able to monitor wildlife accurately, automatically, and remotely in all-weather condition, which lays foundations for applications of wireless image sensor networks in wildlife monitoring.

J. Campbell et al.[?] presented IRISNET, a sensor network architecture that enables the creation of a planetary-scale infrastructure of multimedia sensors that can be shared by a large number of applications. To ensure the efficient collection of sensor readings, IRISNET enables the applicationspecific processing of sensor feeds on the significant computation resources that are typically attached to multimedia sensors. IRISNET enables the storage of sensor readings close to their source by providing a convenient and extensible distributed XML database infrastructure. Finally, IRISNET provides a number of multimedia processing primitives that enable the effective processing of sensor feeds in-network and at-sensor.

\section{PROBLEM FORMULATION}

To boost the transmission speed of video data over WMSNs, our mechanism works on two folds. One is the video tracking and compression. The second is bandwidth balancing routing.

\subsection{Video tracking and compression}

We improved CamShift tracking algorithm based on Histogram Back-projection[?] and Mean Shift Algorithm[?]. The mean shift algorithm works well on static probability distributions but not on dynamic ones. Our approach detects the peak in the probability distribution image by applying mean shift while handling dynamic distributions by readjusting the search window size for the next frame based on the 0th moment of the current frames distribution, which allows the algorithm to anticipate object movement to quickly track the object in the next scene. Specifically, the search area can be restricted around the last known position of the target, resulting in possibly large computational savings. In a single image, the process is iterated until convergence an upper bound on the number of iterations is reached. Apply the detection algorithm to successive frames of a video sequence to track a single target. This type of scheme introduces a feed-back loop, in which the result of the detection is used as input to the next detection process.

In algorithm 1, we choose threshold of convergence to be 1 to get a satisfying tracking result. The location of the search window is used to help track movement.

\subsection{Bandwidth Balancing Routing}

\subsubsection{Basic idea}




\begin{tabular}{l} 
Algorithm 1 Improved CamShift \\
\hline 1: Set the region of interest of the probability distribution \\
image to the entire image \\
2: Select an initial location of the Mean Shift $2 \mathrm{D}$ search \\
window $W$ (scale and location) in the first frame. The \\
selected location is the target distribution to be tracked \\
3: Calculate a color probability distribution of the region \\
centered at the Mean Shift search window, and the re- \\
gion should be slightly bigger than the mean shift search \\
window \\
4: Perform mean shift on the area until suitable conver- \\
gence (T=1). Store the 0th moment (distribution area) \\
and centroid location \\
5: The search window for the next frame is centered around \\
the centroid and the size is scaled by a function of the \\
0th movement; Go to step 3
\end{tabular}

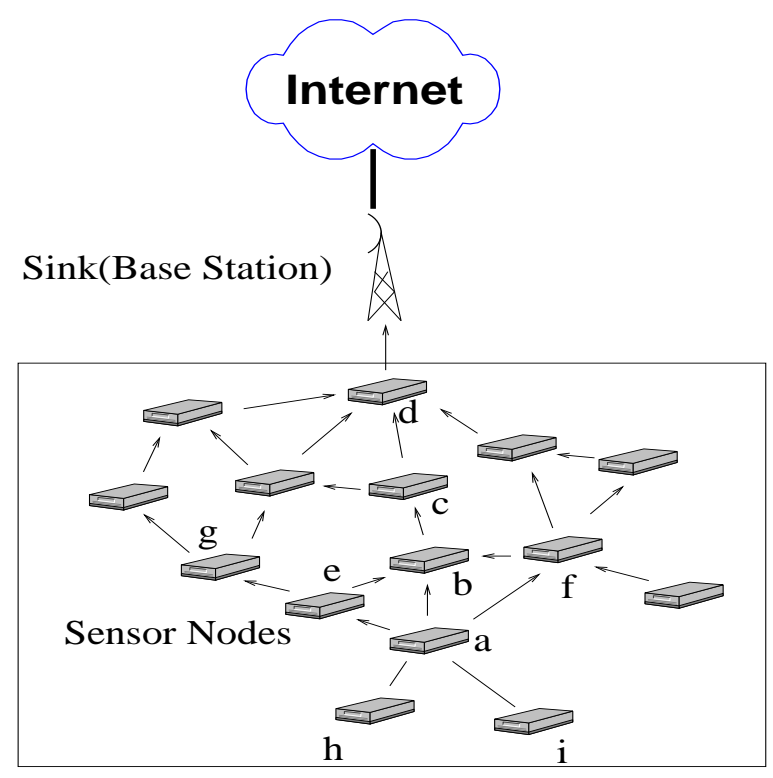

Figure 1: A scenario of WMSN.

In WMSNs, sensor nodes collect video information, process video, and transmit data to sink node(base station). The sink nodes then sends data to Internet. Figure 1 shows an instance of WMSNs. We define the residual bandwidth ability(RBA) for an interface of a node is it possible bandwidth minus the traffic. For Instance, if the possible bandwidth of an interface for a node is $100 \mathrm{MB} / \mathrm{S}$, the traffic is $60 \mathrm{MB} / \mathrm{S}$, then its RBA is $40 \mathrm{MB} / \mathrm{S}$. We use a simple case to illustrate our main idea. Suppose node $a$ collects video data and got it processed. Then it intends to send the data to the sink node. The routing path can be set up by any routing algorithm. Normally, it is fixed when it is set up. The path a-b-c-d can be a routing path. If many other nodes also send data to $b$, and then to the sink node, $b$ has to afford a lot of traffic, while $e$ or $f$ have low traffic. Here comes up with a question, can node $a$ select node $e$ or $f$ as the next hop to avoid busy traffic?

We further assume that the historical RBA vector up to this moment of $b$ is $[\ldots, 85,70,55,40], e$ is $[\ldots, 25,32,35,39]$, and $f$ is $[\ldots, 50,46,42,38]$. A simple strategy will let $a$ select $b$ because it has the highest RBA at the moment. Seemingly, $b$ is the best choice. However, since $b$ is in a hot node serving a high volume traffic and its RBA is going down dramatically, while $e$ or $f$ is serving less traffic and their RBA are going down slowly or even going up, so $a$ should select $e$ or $f$ to avoid hot node and then balance bandwidth utilization.

Our goal is to let each node select the next hop that will have the highest RBA. In our approach, each node can calculate its potential RBA right after this time slot based on its historical and current RBA. In this example, our strategy will let $m_{1}$ choose $R_{2}$.

\subsection{Calculation of Residual Bandwidth Abil- ity}

The communications between nodes in a Wireless Multimedia Sensor Network vary from time to time. It is critical for a node to choose the possible best next hop to transfer video. For each node, the trend of its bandwidth's changing is essential to calculate its potential RBA. Suppose the current time is $k$. A naive method to calculate its potential RBA is as follows. Let the node $N_{i}$ save all its historical and current (time $k$ ) RBA $R_{i_{0}}, R_{i_{1}}, \ldots, R_{i_{k}}$ in a vector. Then it can calculate its potential RBA for time $k+1$ by curve-fitting in numerical analysis that approximates its moving trend. The limitations of this method are to spend too much space to save the RBA values in the vector and too complex for curve-fitting computation.

We propose a statistical based strategy to let each node predict its future RBA. In this scheme, each node only maintains two parameter values. One is its potential energy acceleration and the second is the variance of the acceleration. The two parameters store how its RBA changes. Together with the node's current bandwidth, its future bandwidth capacity can be calculated and is considered its RBA. Thus, each node is able to figure out the best next hop subsequently.

We define the following notations to represent the terms regarding our approach for node $R_{i}$.

$R_{i_{k}}$ : The measured residual bandwidth at time $k$.

$\hat{R_{i_{k}}}$ : The bandwidth predicted at time $k$.

$a_{i_{k}}$ : The acceleration measured at time $k$. It indicates how RBA changes during a time slot.

$\hat{a_{i_{k}}^{-}}$: The acceleration at time $k$ evolved from time $k-1$.

$a_{\hat{i}} \hat{i}_{k}$ : The potential acceleration at time $k$.

$v_{i_{k}}$ : The variance of acceleration updated at time $k$.

$v_{i_{k}}^{-}$: The variance of acceleration at time $k$ evolved from time $k-1$.

$\epsilon$ : The error or noise in the process.

$B_{i_{k}}$ : The blending factor at time $k$.

At time $k$, node $N_{i}$ calls algorithm 2 to calculate future RBA. $N_{i}$ knows its maintained values $\hat{a_{i_{k-1}}}$ and $v_{i_{k-1}}$. It Then it measures its RBA $R_{i_{k}}$ in step 1 . Then it calculate measured acceleration in step 2. $N_{i}$ updates $\hat{a_{i_{k}}^{-}}$and $v_{i_{k}}^{-}$in order to keep its historical RBA to predict its future RBA 

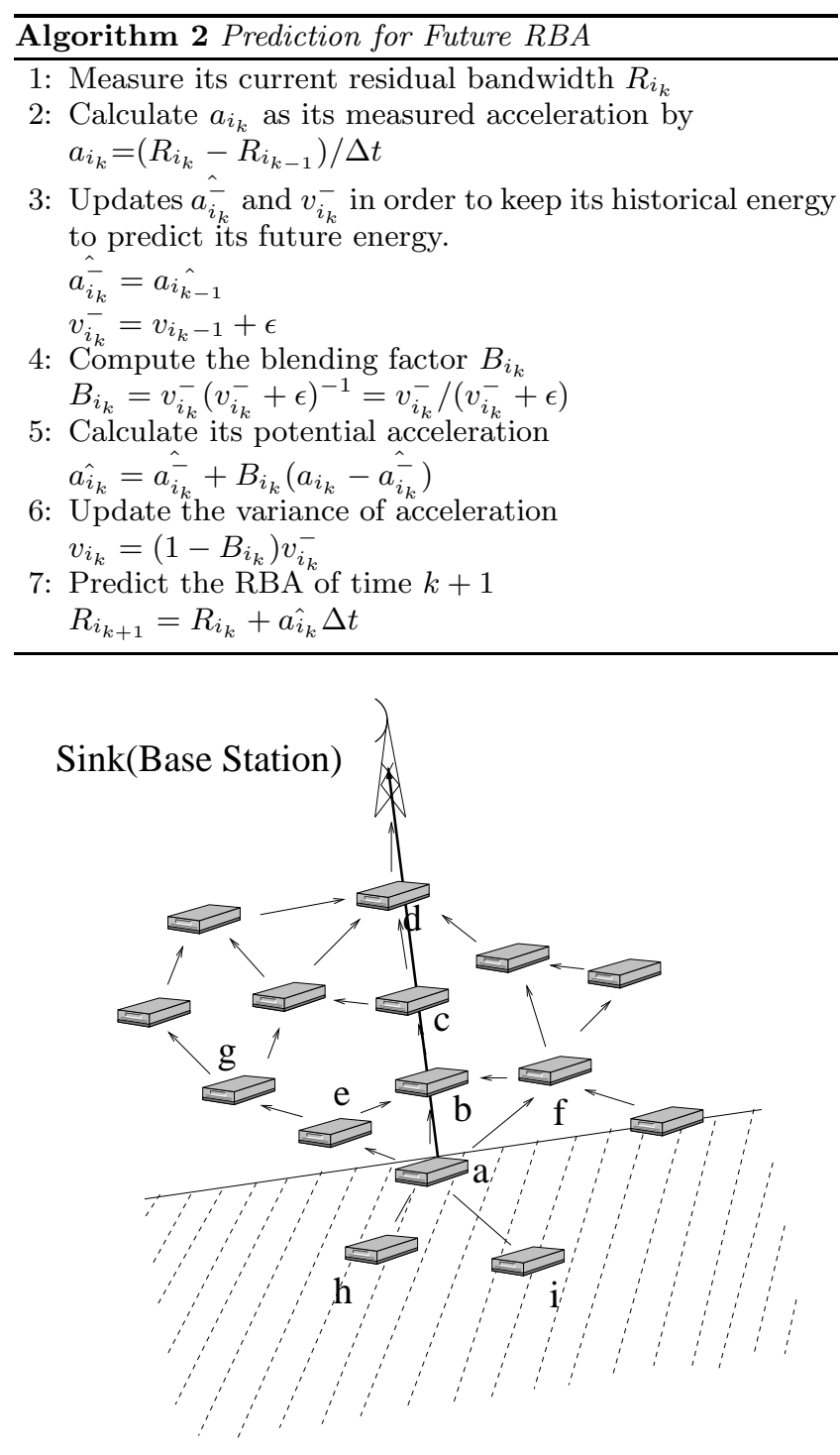

Figure 2: Region for next hop.

in step 3. $N_{i}$ also computes the blending factor $B_{i_{k}}$ in step 4 , which indicates how much the acceleration changes from last time to current time. Once $N_{i}$ obtains the blending factor $B_{i_{k}}$ and the evolved acceleration $\hat{a_{i_{k}}^{-}}$, it knows how much the acceleration changes and the evolved acceleration. Additionally, $R_{i}$ considers the measured acceleration $a_{i_{k}}$. Then it calculates its potential acceleration in step 5. This acceleration will be used to predict its RBA of time $k+1$. It updates the variance of acceleration for future utilization in step 6 . It predicts its future RBA of time $k+1$ in step 7 .

\subsection{Region for next hop}

When a node $N_{i}$ intends to transfer video data to the sink node, it selects the neighbor node with highest potential RBA as its next hop. Apparently, $N_{i}$ will not select any nodes in the opposite direction from $N_{i}$ to the sink node.

How does node $N_{i}$ figure out the region where next hop falls? In current WMSNs, each device is equipped with GPS and hence it knows its location. We assume that the sender knows its location and the location of the receiver. The assumption is very common in geographic routing[?]. Figure 2 shows the scenario. Suppose node $a$ intends to send video data to the sink node, it creates a ray by connecting itself with the sink. Then it draws a line perpendicular to the ray. Then the region on the same side of the sink is available region for next hop. As in figure 2, the dash line region is not available. That is, $a$ will never select a node in that area for next hop.

\subsection{Bandwidth Balancing Routing}

Once a node $N_{i}$ intends to send video data to the sink, it needs to select the node with highest RBA as the next hop.It sends a signal to its neighbors in the available region. Upon receiving the signal, each receiver calls algorithm 2 to calculate its RBA of next time and returns the RBA to node $N_{i}$. When node $N_{i}$ receives all RBAs, it calls Selection to select the one with highest RBA as the next hop.

\section{PERFORMANCE EVALUATION}

We evaluated our mechanism in a simulated noiseless radio network environment by MATLAB. We create a topology that consists of a number of randomly distributed nodes. A sink node is located in the edge of the area. We performed a sequence of experiments in which the number of nodes varies from 50 to 800 with increment of 50 . For each number of mobile users, we measure size of data the sink node received 10 times and present the average. In WMSNs, the life time of a relay station is important. If one node runs turns down, there will be a hole[?] and the whole throughput will be decreased dramatically. So we also measure how many alive nodes long with the time elapsing.

Our approach considers balancing bandwidth utilization by RBA selection. We compare our approach("RBA") with LEACH and GPSR.

Fig. 3 shows our evaluation of data received in sink node. Among the three approaches, the scheme GPSR has the lowest throughput. Our approach RBA generate highest received data. This indicates that bandwidth balance can improve aggregate data throughput for the sink because the traffic are allocated more reasonable to the sink.

Fig. ?? shows how many alive nodes of the three schemes with time elapsing. We initialize 800 nodes and ask them to send data to the sink. We measure how many alive nodes from the 5th second to the 80th second with increment of 5. the GPSR results in the lowest average lift time. That is because GPSR adopts a landmark node to forward a lot of data when there is a hole. However, the landmark is easy to turn down with high volume traffic. Then the hole is bigger [?]. Our approach maintains the largest alive nodes because we always balance bandwidth and then makes the life time longer.

\section{CONCLUSION}

In this paper, we present a RBA based bandwidth balancing mechanism to transfer video data in Wireless Multimedia Sensor Networks. We first present an improved CamShift to comrpess video for video tracking and transmission. Then 


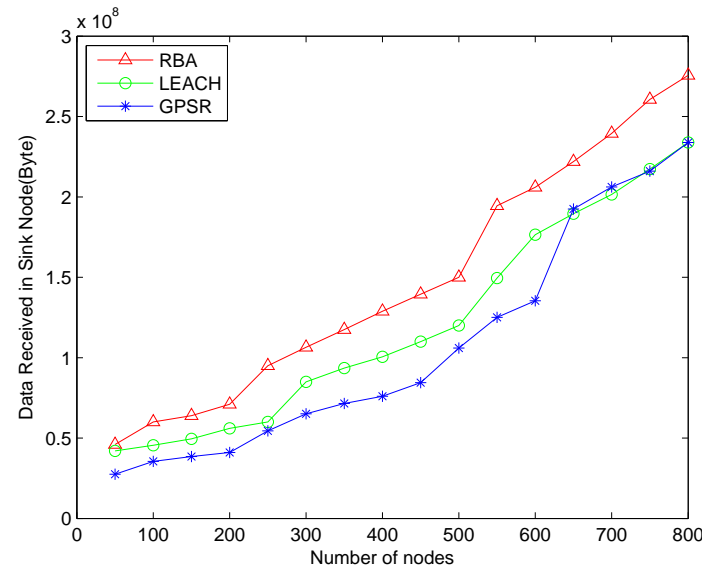

we propose a statistical based mechanism to balance bandwidth utilization.In the approach, each node only needs to remain two factor to store its historical residual bandwidth ability(RBA) trend and then it is able to predict its bandwidth of next time. Each node selects the next hop with potential highest RBA to relay data transmission. Simulations demonstrate that our approach results in higher data received by sink node in the network and longer average life time of relay stations over related approaches.

Figure 3: Aggregate throughput

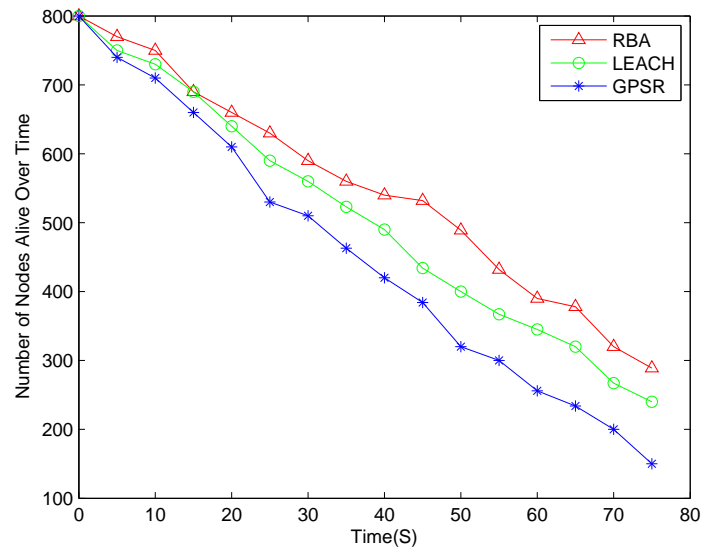

Figure 4: Aggregate throughput 


\section{Compression of Video Tracking and Bandwidth Balancing Routing in Wireless Multimedia Sensor Networks}

\author{
Yin Wang Department of \\ Mathematics and Computer \\ Science \\ Lawrence Technological \\ University \\ ywang12@ltu.edu
Juan Guo Department of Computer Science and Information Systems
University of North Georgia juan.guo@ung.edu

\author{
Jianjun Yang Department of \\ Computer Science and \\ Information Systems \\ University of North Georgia \\ jianjun.yang@ung.edu
}

\author{
Ju Shen Department of \\ Computer Science \\ University of Dayton \\ jushen.tom@gmail.com
}

\begin{abstract}
There has been a tremendous growth in multimedia applications over wireless networks. Wireless Multimedia Sensor Networks(WMSN) have become the premier choice in many research communities and industry. Many state-to-art applications, such as surveillance, traffic monitoring, and remote heath care are essentially video tracking and transmission in WMSN. The transmission speed is constrained by big size of video data and fixed bandwidth allocation in fixed routing path. In this paper, we present a CamShift algorithm to compress the tracking of videos. Then we propose a bandwidth balancing strategy in which the sensor nodes are able to dynamically select the node for next hop with the highest potential bandwidth capacity to resume communication. Key to the strategy is that each node merely maintains two parameters that contains the historical network traffic and then calculates its bandwidth capacity. Then each node selects the next hop with the highest potential bandwidth capacity. Simulations demonstrate that our approach significantly increase the aggregate throughput and decrease the latency on video transmission in Wireless Multimedia Sensor Network environment.
\end{abstract}

\section{Keywords}

CamShift, bandwidth balancing, network traffic

\section{INTRODUCTION}

Wireless Multimedia Sensor Networks(WMSN) have emerged as one of the key technologies for wireless communications. They are undergoing rapid development and have inspired numerous applications because of their advantages. More

\author{
Kun Hua Department of \\ Electrical and Computer \\ Engineering \\ Lawrence Technological \\ University \\ khua@ltu.edu
}

recently, the availability of inexpensive hardware such as CMOS cameras and microphones that are able to ubiquitously capture multimedia content from the environment has fostered the development of Wireless Multimedia Sensor Networks (WMSNs), which are networks of wirelessly interconnected devices that allow retrieving video and audio streams, still images, and scalar sensor data[?].

Wireless multimedia sensor networks will enable several new applications[?], which include: First, Surveillance.Video and audio sensors will be used to enhance and complement existing surveillance systems against crime and terrorist attacks. Large scale networks of video sensors can extend the ability of law-enforcement agencies to monitor areas, public events, private properties, and borders. Second, Traffic Monitoring and Enforcement.It will be possible to monitor car traffic in big cities or highways and deploy services that offer traffic routing advice to avoid congestion.Third, Personal and Health Care. Multimedia sensor networks can be used to monitor and study the behavior of people as a means to identify the causes of illnesses. Fourth, Environmental and Industrial.Several projects on habitat monitoring that use acoustic and video feeds are being envisaged, in which information has to be conveyed in a time-critical fashion.

Despite of the fact that wireless bandwidth has been increasing significantly, from a theoretical limit of $11 \mathrm{Mbps}$ for $802.11 \mathrm{~b}$ to $54 \mathrm{Mbps}$ for $802.11 \mathrm{~g}$, and to $540 \mathrm{Mbps}$ for $802.11 \mathrm{n}$, there has always been high bandwidth demand resulting from multimedia data[?]. Moreover, the routing path from a node to the sink node is normally created by a kind of routing protocol and the path is fixed. Hence some nodes are affording busy traffic but other nodes that are not in the routing path are idle. In this paper, we developed a novel compression video tracking and bandwidth balancing mechanism to boost the video transmission speed in WMSNs. Our contributions are two folds. First, we present a CamShift algorithm to compress tracking of videos. Then we propose a bandwidth balancing strategy in which the sensor nodes are able to dynamically select the node for next hop with the highest potential bandwidth capacity to re- 
sume communication. Key to the strategy is that each node merely maintains two parameters that contains the historical network traffic and then calculates its bandwidth capacity. Then each node selects the next hop with the highest potential bandwidth capacity. Our approach significantly increase the aggregate throughput and decrease the latency on video transmission in Wireless Multimedia Sensor Network environment.

The rest of the paper is organized as follows. Section II discusses the related research on this topic. Section III proposes a novel method that select the best relay station. We evaluate the proposed schemes by simulations and describe the performance results in Section IV. Section V concludes the paper.

\section{RELATED WORK}

Various approaches regarding video processing and transmission over WMSNs were proposed. Q. Cai et al. [?, ?] proposed a dynamic action recognition, which is important to capture videos in WMSNs. In their approach, a Dynamic Structure Preserving Map is proposed to effectively recognize objects' actions in video sequences. They modified and improved the adaptive learning procedure in self-organizing map (SOM) to capture dynamics of best matching neurons through Markov random walk. The method can learn implicit spatial-temporal correlations from sequential action feature sets and preserve the intrinsic topologies characterized by different human motions. The mechanism is able to learn low-level features in challenging video data. The projection from high dimensional action features to low dimensional latent neural distribution significantly reduces the computational cost and data redundancy in the recognition process.

Ahmed et al. [?] studied to improve the quality of MPEG-4 transmission on wireless using Differentiated Services. They investigated QoS provisioning between MPEG-4 video application and Diffserv networks. To achieve the best possible QoS, all the components involved in the transmission process must collaborate. For example, the server must use stream properties to describe the QoS requirement for each stream to the network. They propose a solution by distinguishing the video data into important video data and less important video data. Packets marked as less important are dropped in the first case if there is any congestion, so that the receiver can regenerate the video with the received important information.

Budagavi et al. [?] improved the performance of video over wireless channels by multiframe video coding. The multiframe coder uses the redundancy that exists across multiple frames in a typical video conferencing sequence so that additional compression can be achieved using their MF-BMC (Multi Frame - Block Motion Compensation) approach. They modeled the error propagation using the Markov chain, and concluded that use of multiple frames in motion increases the robustness. Their proposed MF-BMC scheme has been shown to be more robust on wireless networks when compared to the base-level H.263 codec which uses SF-BMC (Single Frame-BMC).

J. Zhang et al.[?, ?] developed a wildlife monitoring system based on wireless image sensor networks. In their study, system architecture of the wildlife monitoring system based on the wireless image sensor networks was presented to overcome the shortcomings of the traditional monitoring methods. Specifically, some key issues including design of wireless image sensor nodes and software process design have been studied and presented. A self-powered rotatable wireless infrared image sensor node and an aggregation node designed for large amounts of data were developed. In addition, their corresponding software was designed. The proposed system is able to monitor wildlife accurately, automatically, and remotely in all-weather condition, which lays foundations for applications of wireless image sensor networks in wildlife monitoring.

J. Campbell et al.[?] presented IRISNET, a sensor network architecture that enables the creation of a planetary-scale infrastructure of multimedia sensors that can be shared by a large number of applications. To ensure the efficient collection of sensor readings, IRISNET enables the applicationspecific processing of sensor feeds on the significant computation resources that are typically attached to multimedia sensors. IRISNET enables the storage of sensor readings close to their source by providing a convenient and extensible distributed XML database infrastructure. Finally, IRISNET provides a number of multimedia processing primitives that enable the effective processing of sensor feeds in-network and at-sensor.

\section{PROBLEM FORMULATION}

To boost the transmission speed of video data over WMSNs, our mechanism works on two folds. One is the video tracking and compression. The second is bandwidth balancing routing.

\subsection{Video tracking and compression}

We improved CamShift tracking algorithm based on Histogram Back-projection[?] and Mean Shift Algorithm[?]. The mean shift algorithm works well on static probability distributions but not on dynamic ones. Our approach detects the peak in the probability distribution image by applying mean shift while handling dynamic distributions by readjusting the search window size for the next frame based on the 0th moment of the current frames distribution, which allows the algorithm to anticipate object movement to quickly track the object in the next scene. Specifically, the search area can be restricted around the last known position of the target, resulting in possibly large computational savings. In a single image, the process is iterated until convergence an upper bound on the number of iterations is reached. Apply the detection algorithm to successive frames of a video sequence to track a single target. This type of scheme introduces a feed-back loop, in which the result of the detection is used as input to the next detection process.

In algorithm 1, we choose threshold of convergence to be 1 to get a satisfying tracking result. The location of the search window is used to help track movement.

\subsection{Bandwidth Balancing Routing}

\subsubsection{Basic idea}




\begin{tabular}{l} 
Algorithm 1 Improved CamShift \\
\hline 1: Set the region of interest of the probability distribution \\
image to the entire image \\
2: Select an initial location of the Mean Shift $2 \mathrm{D}$ search \\
window $W$ (scale and location) in the first frame. The \\
selected location is the target distribution to be tracked \\
3: Calculate a color probability distribution of the region \\
centered at the Mean Shift search window, and the re- \\
gion should be slightly bigger than the mean shift search \\
window \\
4: Perform mean shift on the area until suitable conver- \\
gence (T=1). Store the 0th moment (distribution area) \\
and centroid location \\
5: The search window for the next frame is centered around \\
the centroid and the size is scaled by a function of the \\
0th movement; Go to step 3
\end{tabular}

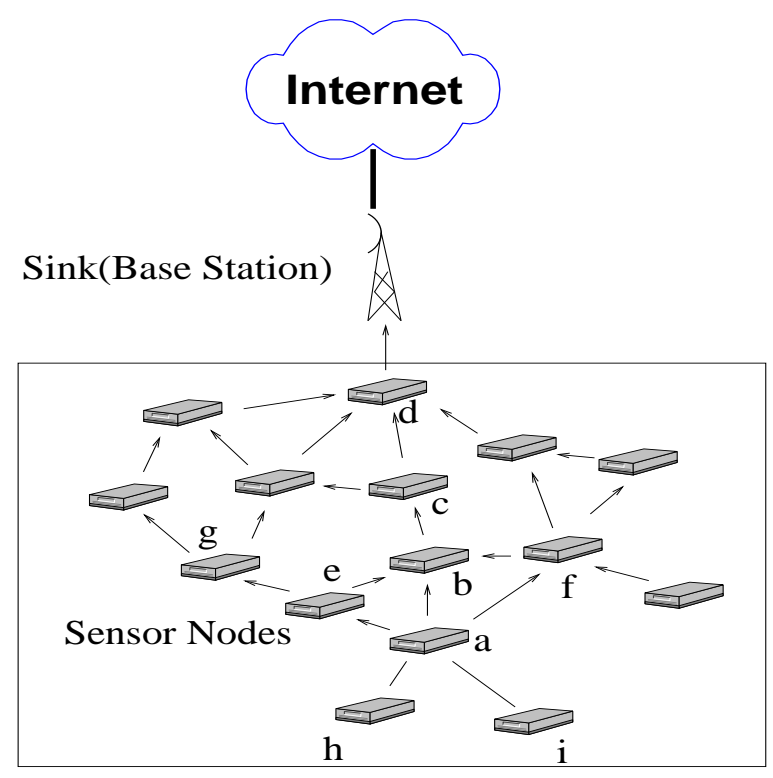

Figure 1: A scenario of WMSN.

In WMSNs, sensor nodes collect video information, process video, and transmit data to sink node(base station). The sink nodes then sends data to Internet. Figure 1 shows an instance of WMSNs. We define the residual bandwidth ability(RBA) for an interface of a node is it possible bandwidth minus the traffic. For Instance, if the possible bandwidth of an interface for a node is $100 \mathrm{MB} / \mathrm{S}$, the traffic is $60 \mathrm{MB} / \mathrm{S}$, then its RBA is $40 \mathrm{MB} / \mathrm{S}$. We use a simple case to illustrate our main idea. Suppose node $a$ collects video data and got it processed. Then it intends to send the data to the sink node. The routing path can be set up by any routing algorithm. Normally, it is fixed when it is set up. The path a-b-c-d can be a routing path. If many other nodes also send data to $b$, and then to the sink node, $b$ has to afford a lot of traffic, while $e$ or $f$ have low traffic. Here comes up with a question, can node $a$ select node $e$ or $f$ as the next hop to avoid busy traffic?

We further assume that the historical RBA vector up to this moment of $b$ is $[\ldots, 85,70,55,40], e$ is $[\ldots, 25,32,35,39]$, and $f$ is $[\ldots, 50,46,42,38]$. A simple strategy will let $a$ select $b$ because it has the highest RBA at the moment. Seemingly, $b$ is the best choice. However, since $b$ is in a hot node serving a high volume traffic and its RBA is going down dramatically, while $e$ or $f$ is serving less traffic and their RBA are going down slowly or even going up, so $a$ should select $e$ or $f$ to avoid hot node and then balance bandwidth utilization.

Our goal is to let each node select the next hop that will have the highest RBA. In our approach, each node can calculate its potential RBA right after this time slot based on its historical and current RBA. In this example, our strategy will let $m_{1}$ choose $R_{2}$.

\subsection{Calculation of Residual Bandwidth Abil- ity}

The communications between nodes in a Wireless Multimedia Sensor Network vary from time to time. It is critical for a node to choose the possible best next hop to transfer video. For each node, the trend of its bandwidth's changing is essential to calculate its potential RBA. Suppose the current time is $k$. A naive method to calculate its potential RBA is as follows. Let the node $N_{i}$ save all its historical and current (time $k$ ) RBA $R_{i_{0}}, R_{i_{1}}, \ldots, R_{i_{k}}$ in a vector. Then it can calculate its potential RBA for time $k+1$ by curve-fitting in numerical analysis that approximates its moving trend. The limitations of this method are to spend too much space to save the RBA values in the vector and too complex for curve-fitting computation.

We propose a statistical based strategy to let each node predict its future RBA. In this scheme, each node only maintains two parameter values. One is its potential energy acceleration and the second is the variance of the acceleration. The two parameters store how its RBA changes. Together with the node's current bandwidth, its future bandwidth capacity can be calculated and is considered its RBA. Thus, each node is able to figure out the best next hop subsequently.

We define the following notations to represent the terms regarding our approach for node $R_{i}$.

$R_{i_{k}}$ : The measured residual bandwidth at time $k$.

$\hat{R_{i_{k}}}$ : The bandwidth predicted at time $k$.

$a_{i_{k}}$ : The acceleration measured at time $k$. It indicates how RBA changes during a time slot.

$\hat{a_{i_{k}}^{-}}$: The acceleration at time $k$ evolved from time $k-1$.

$a_{\hat{i}} \hat{i}_{k}$ : The potential acceleration at time $k$.

$v_{i_{k}}$ : The variance of acceleration updated at time $k$.

$v_{i_{k}}^{-}$: The variance of acceleration at time $k$ evolved from time $k-1$.

$\epsilon$ : The error or noise in the process.

$B_{i_{k}}$ : The blending factor at time $k$.

At time $k$, node $N_{i}$ calls algorithm 2 to calculate future RBA. $N_{i}$ knows its maintained values $\hat{a_{i_{k-1}}}$ and $v_{i_{k-1}}$. It Then it measures its RBA $R_{i_{k}}$ in step 1 . Then it calculate measured acceleration in step 2. $N_{i}$ updates $\hat{a_{i_{k}}^{-}}$and $v_{i_{k}}^{-}$in order to keep its historical RBA to predict its future RBA 

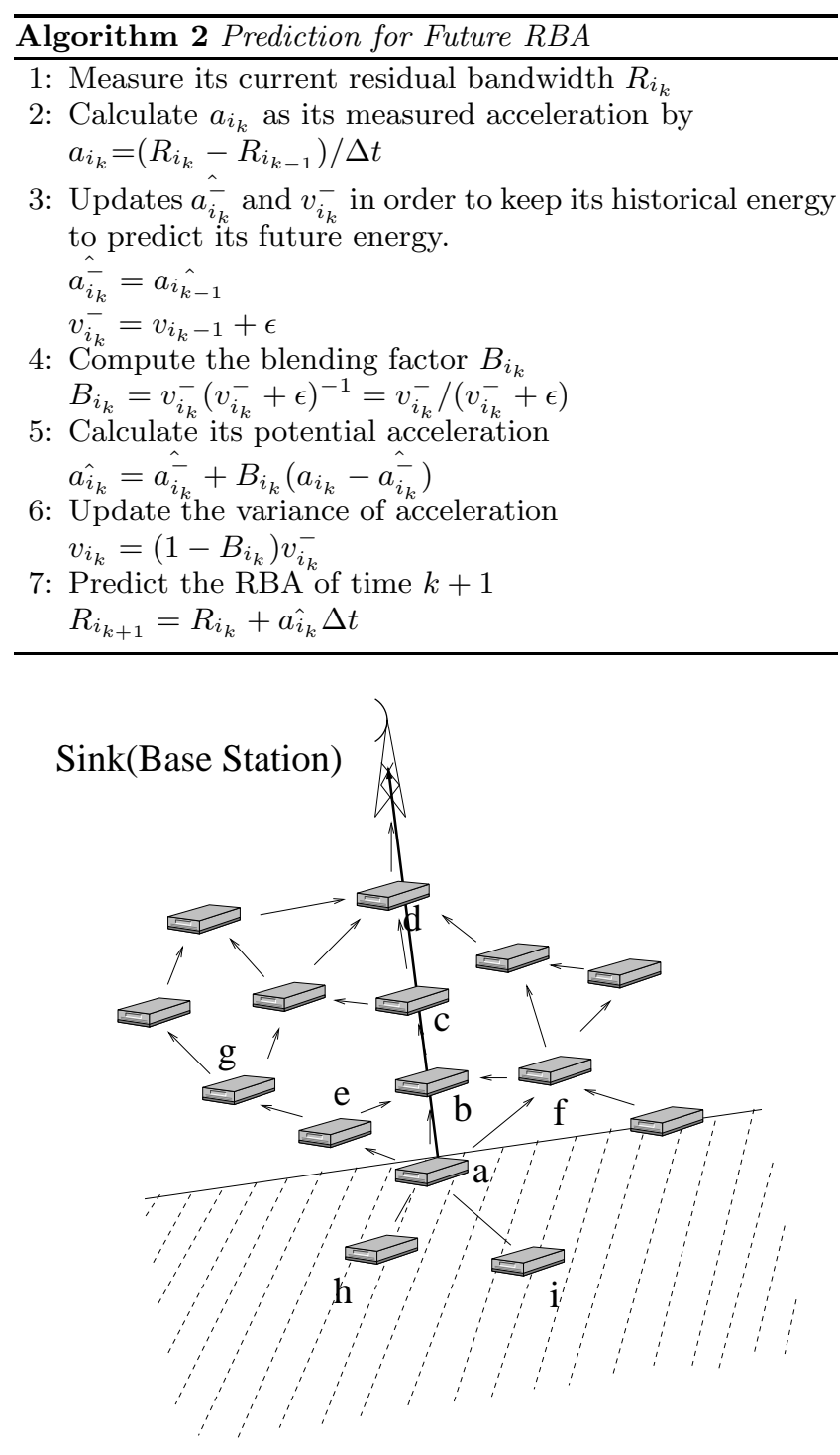

Figure 2: Region for next hop.

in step 3. $N_{i}$ also computes the blending factor $B_{i_{k}}$ in step 4 , which indicates how much the acceleration changes from last time to current time. Once $N_{i}$ obtains the blending factor $B_{i_{k}}$ and the evolved acceleration $\hat{a_{i_{k}}^{-}}$, it knows how much the acceleration changes and the evolved acceleration. Additionally, $R_{i}$ considers the measured acceleration $a_{i_{k}}$. Then it calculates its potential acceleration in step 5. This acceleration will be used to predict its RBA of time $k+1$. It updates the variance of acceleration for future utilization in step 6 . It predicts its future RBA of time $k+1$ in step 7 .

\subsection{Region for next hop}

When a node $N_{i}$ intends to transfer video data to the sink node, it selects the neighbor node with highest potential RBA as its next hop. Apparently, $N_{i}$ will not select any nodes in the opposite direction from $N_{i}$ to the sink node.

How does node $N_{i}$ figure out the region where next hop falls? In current WMSNs, each device is equipped with GPS and hence it knows its location. We assume that the sender knows its location and the location of the receiver. The assumption is very common in geographic routing[?]. Figure 2 shows the scenario. Suppose node $a$ intends to send video data to the sink node, it creates a ray by connecting itself with the sink. Then it draws a line perpendicular to the ray. Then the region on the same side of the sink is available region for next hop. As in figure 2, the dash line region is not available. That is, $a$ will never select a node in that area for next hop.

\subsection{Bandwidth Balancing Routing}

Once a node $N_{i}$ intends to send video data to the sink, it needs to select the node with highest RBA as the next hop.It sends a signal to its neighbors in the available region. Upon receiving the signal, each receiver calls algorithm 2 to calculate its RBA of next time and returns the RBA to node $N_{i}$. When node $N_{i}$ receives all RBAs, it calls Selection to select the one with highest RBA as the next hop.

\section{PERFORMANCE EVALUATION}

We evaluated our mechanism in a simulated noiseless radio network environment by MATLAB. We create a topology that consists of a number of randomly distributed nodes. A sink node is located in the edge of the area. We performed a sequence of experiments in which the number of nodes varies from 50 to 800 with increment of 50 . For each number of mobile users, we measure size of data the sink node received 10 times and present the average. In WMSNs, the life time of a relay station is important. If one node runs turns down, there will be a hole[?] and the whole throughput will be decreased dramatically. So we also measure how many alive nodes long with the time elapsing.

Our approach considers balancing bandwidth utilization by RBA selection. We compare our approach("RBA") with LEACH and GPSR.

Fig. 3 shows our evaluation of data received in sink node. Among the three approaches, the scheme GPSR has the lowest throughput. Our approach RBA generate highest received data. This indicates that bandwidth balance can improve aggregate data throughput for the sink because the traffic are allocated more reasonable to the sink.

Fig. ?? shows how many alive nodes of the three schemes with time elapsing. We initialize 800 nodes and ask them to send data to the sink. We measure how many alive nodes from the 5th second to the 80th second with increment of 5. the GPSR results in the lowest average lift time. That is because GPSR adopts a landmark node to forward a lot of data when there is a hole. However, the landmark is easy to turn down with high volume traffic. Then the hole is bigger [?]. Our approach maintains the largest alive nodes because we always balance bandwidth and then makes the life time longer.

\section{CONCLUSION}

In this paper, we present a RBA based bandwidth balancing mechanism to transfer video data in Wireless Multimedia Sensor Networks. We first present an improved CamShift to comrpess video for video tracking and transmission. Then 


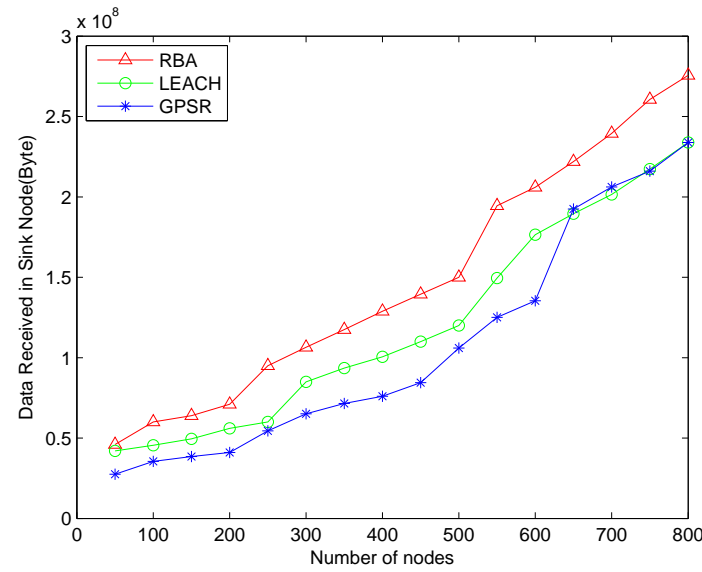

we propose a statistical based mechanism to balance bandwidth utilization.In the approach, each node only needs to remain two factor to store its historical residual bandwidth ability(RBA) trend and then it is able to predict its bandwidth of next time. Each node selects the next hop with potential highest RBA to relay data transmission. Simulations demonstrate that our approach results in higher data received by sink node in the network and longer average life time of relay stations over related approaches.

Figure 3: Aggregate throughput

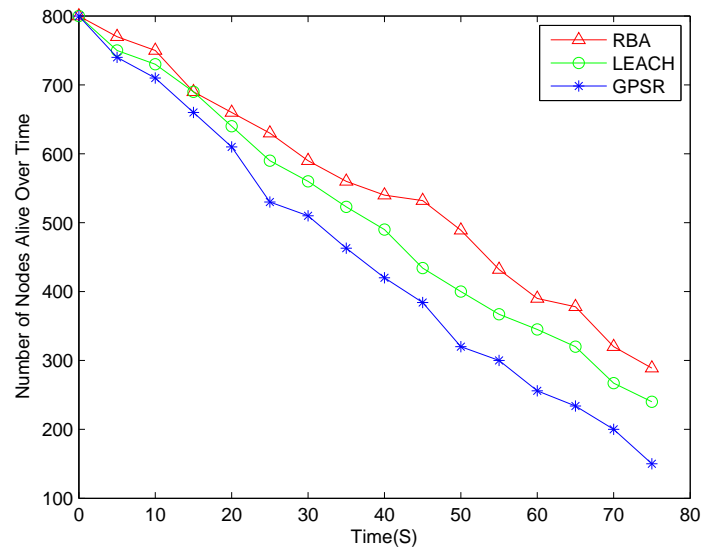

Figure 4: Aggregate throughput 